\title{
Utility of Video-Fundoscopy and Prospects of Portable Stereo-Photography of The Ocular Fundus in Neurological Patients
}

Tigran Khachatryan ( $\square$ tigrank@hs.uci.edu )

University of California, Irvine

Tahseen Mozaffar

University of California, Irvine

Lilit Mnatsakanyan

University of California, Irvine

\section{Research Article}

Keywords: Fundoscopy, ophthalmoscope, smartphone, 3D, fundus photography

Posted Date: October 25th, 2021

DOI: https://doi.org/10.21203/rs.3.rs-881801/v1

License: (1) This work is licensed under a Creative Commons Attribution 4.0 International License.

Read Full License

Version of Record: A version of this preprint was published at BMC Neurology on February 19th, 2022. See the published version at https://doi.org/10.1186/s12883-022-02578-5. 

Utility of Video-fundoscopy and Prospects of Portable Stereo-photography of the Ocular Fundus in
Neurological Patients

Tigran Khachatryan MD, $\mathrm{PhD}^{1}$, Tahseen Mozaffar, $\mathrm{MD}^{1,2}$, Lilit Mnatsakanyan, $\mathrm{MD}^{1}$ Department of Neurology ${ }^{1}$ and Pathology \& Laboratory Medicine, ${ }^{2}$

University of California, Irvine

Address Correspondence to:

Tigran Khachatryan, MD PhD

Department of Neurology

University of California, Irvine

200 S. Manchester Avenue, Ste. 206

Orange, CA 92868.

Tel: 714-456-7352

Fax: 714-456-8805

Email: tigrank@hs.uci.edu

Keywords: Fundoscopy, ophthalmoscope, smartphone, 3D, fundus photography

Acknowledgments: None

Word Counts

Title: 15 words (118 characters)

Abstract: 233 words

Manuscript: 1389 words

Figures: 4

Tables: 0

References: 17 


\section{Abstract \\ Background}

Proper evaluation of ocular fundi is an integral part of neurological examination. Unfortunately, neurology residents are increasingly uncomfortable performing fundoscopy and interpreting findings because of diminishing skills and lack of experience. This became more prominent during the COVID-19 pandemic as fundoscopy requires proximity to the patient.

With the recent dramatic improvement of smartphone cameras, fundus photography using PanOptic Ophthalmoscope (Welch Allyn, Skaneateles Falls, NY) with a smartphone adapter offered an alternative to direct fundoscopic examination. Having not found a good solution in the market, we created our own design of a universal smartphone adapter.

\section{Results}

Here we present our initial experience of fundus video/photography in patients, who presented with encephalopathy, headache, seizure, vision loss, and other ocular symptoms. Fundoscopic abnormalities were discovered in 11 out of 100 patients. Some were incidental findings and were unrelated to the presentation. In one case, fundoscopy played a critical role in establishing the correct diagnosis.

\section{Conclusions}

Our custom-designed smartphone adapter allowed obtaining high-quality video and photo recordings using PanOptic Ophthalmoscope. The acquisition of high-quality photos enables a high-yield diagnostic tool and allows revisiting the image in the patient's chart. Improvement of smartphone cameras opens vast horizons for stereo-fundoscopy and 3D reconstruction of the ocular fundus without using sophisticated and costly equipment. Microscopic eye movements allow taking snapshots of two side-by-side photos for 3D reconstruction and stereoscopic image viewing, which is the next level of optic disc assessment.

\section{Background}


Fundoscopic examination is a basic competency requirement for all Neurologists and an important component of neurological examination $[1,2]$. Traditional direct fundoscopy requires that examiners position themselves close to the patient which became a serious limitation during COVID-19 pandemic.[3] As part of a resident quality improvement project, medical records of 100 consecutive patients, admitted to the Department of Neurology at UC Irvine Medical Center during the pandemic, were retrospectively reviewed. None of the patients underwent a fundoscopic examination.

With dramatic improvement of camera quality in modern smartphones, fundus photography using PanOptic Ophthalmoscope (Welch Allyn, Skaneateles Falls, NY) with a smartphone adapter offered an alternative to direct examination of ocular fundus. Having not found a good solution in the market, we explored the idea of creating our very own design of universal smartphone adapter for PanOptic Ophthalmoscope to fit all smartphone types, which would help reducing very close contact with the patient and reduce the time needed for examination.

\section{Methods}

This work was conducted as part of resident quality improvement project at UC Irvine Medical Center. : All methods were performed in accordance with the relevant guidelines and regulations.

The adapter was designed using MeshMixer (Autodesk Inc.) 3D modeling software (Figure 1-a). It was then printed with Polyethylene terephthalate glycol filament on a 3D Fused Deposition Modeling printer.

The attachment consists of a foldable arm, a flat surface with double-sided adhesive and an ocular piece, which fits exactly the ocular part of PanOptic Ophthalmoscope (Figure 1-c). The adapted can be permanently attached to any smartphone case and, with the foldable arm, can be seamlessly carried in the pocket (Figure 1-b). Additionally, we designed a smartphone powered light source, which eliminated the need for charging and carrying the battery piece (Figure 1-d). Thus, one needs to carry only the head of PanOptic ophthalmoscope to obtain fundoscopic images. 
Patients, presenting to the emergency department with complaints of headache, ocular symptoms (visual disturbances, blindness, diplopia, pain), seizures or encephalopathy underwent fundoscopic videography and/or photography over the period from December 2019 to February 2021. We did not use pharmacological pupillary dilation for any of the patients. Images were captured using iPhone 11 Pro (Apple Inc, CA, USA) smartphone. Photographs were directly uploaded to patient's chart on Epic using Haiku app for IOS (Epic systems corporation, Verona, WI). Video data was de-identified and deleted after subtracting all necessary snapshots. Fundus videographs and photographs were then reviewed by on-call senior resident and later by the attending neurologist on service during formal rounds.

We also developed a methodology of capturing stereoscopic images of the optic disc using a single smartphone camera. Healthy volunteer subjects were asked to look sequentially at two objects or light sources located 6 feet away from the eye and 30 centimeters apart from each other. Video fundoscopy was performed while the subject was performing the task. The camera remained static in respect to the subject's forehead, but eyeball moved in the horizontal plane, providing visualization of the fundus under two different angles equal to ten degrees. Subsequently, snapshots from each respective position were obtained, which essentially represented a pair of stereoscopic images. Google cardboard or similar 3D headset was utilized to view stereoscopic images.

Upon admission to the hospital, all patients or their legal representatives signed admission terms and conditions agreement, which includes statements about obtaining medical video/photographs. Furthermore, fundoscopy is a part of neurological exam and does not require separate written consent. There are no identifiable features in presented group of photographs.

\section{Results:}

Data was analyzed after performing fundus video/photographs on 100 patients. The presentation included encephalopathy in $20 \%$, headache in $33 \%$, seizure in $16 \%$, vision loss in $12 \%$ and other ocular symptoms (diplopia, decreased acuity of vision, eye pain) in $19 \%$ of patients. The mean age of the examinees was 44.7 years (range 20-89). 
Fundoscopic abnormalities were discovered in 11 patients, although some were incidental findings as these were unrelated to the presentation or chief complaint (Figure $2 \mathrm{a}-\mathrm{g}$ ). In one patient, fundoscopy played a critical role in establishing the diagnosis.

A 78 yo man with history of uncontrolled diabetes and chemotherapy due to gastric adenocarcinoma was brought in by family due to altered mental status and decreased oral intake. On initial evaluation patient was diagnosed with diabetic keto acidosis due to beta hydroxybutyrate level of $>4.0 \mathrm{mmol} / \mathrm{L}$, bicarbonate level of $19 \mathrm{mmol} / \mathrm{L}$ and anion gap of $16 \mathrm{mmol} / \mathrm{L}$ prompting admission to medical ICU for further care. Neurology was consulted due to history of bilateral lower extremity weakness after a chiropractic manipulation. Fundoscopy was performed as part of the neurological examination and showed bilateral grade 2 papilledema (Figure 3). Based on the findings lumbar puncture was recommended which revealed opening pressure of $55 \mathrm{~cm} \mathrm{H} 2 \mathrm{O}$ and 235 nucleated cells. Final cytological diagnosis of leptomeningeal carcinomatosis due to gastric adenocarcinoma was confirmed by CDX2 immunostaining. Patient underwent VP shunt placement and was discharged to acute rehabilitation facility with outpatient follow-up.

The aforementioned methodology of stereoscopic imaging was developed relatively late in the study, thus was not routinely utilized in presented group of patients. After finalizing the technique on two healthy volunteers (Figure 4a and 4b), it was tested on one patient with normal fundoscopic findings. Stereoscopic images resulted in subjectively better perception of image depth, easier estimation of cup to disc ratio and enhanced visualization of the vessels in relation to the optic disc (Figure 4c).

\section{Discussion:}

Since first direct visualization of ocular fundus by Hermann von Helmholtz in 1851, fundoscopy has become an integral part of neurological examination.[4-6] Many authors have emphasized the importance of proper fundoscopic examination in neurological patients. [6, 7] However, with introduction of neuroimaging modalities such as CT and MRI, non-ophthalmology specialists are having an increasingly difficult time to perform fundoscopy or interpret the findings because of diminishing skills and lack of experience. [4, 6-9] This is partly explained by the necessity to dilate the pupil for adequate examination, which inherently 
makes serial neurological examinations more difficult. In 1886, Jackman and Webster published the first human fundus photograph, beginning a new era in visualization of ocular fundus. $[5,9,10]$ With advancement of technology, non-mydriatic fundus cameras came into practice.[4, 11] The wide field of view and possibility of performing the examination without pharmacologic dilation of the pupil became a huge advantage of non-mydriatic fundus cameras.[4,12] It has a number of other advantages, including higher rate of successful visualization, possibility to share the image among multiple providers, avoiding repeated examinations causing patient discomfort as well as storing images for comparison in the future. [4, $7,13,14]$ Several studies have shown that images even can be obtained by non-medical personnel after a short training period, unlike direct fundoscopy, when visualization and interpretation occur simultaneously.[2, 3, 5, 7, 11]

However, there is a host of limitations to non-mydriatic fundus photography. Even handheld nonmydriatic cameras are bulky and difficult to carry around at all times and are not widely used outside the ophthalmology clinic settings.[15] The cost of such cameras is another limitation of its universal use, particularly in countries with limited resources.[7] Additionally, direct ophthalmoscopy allows visualization of retinal venous pulsation, while such interpretation is impossible by viewing still photographs. [16] Finally, trying to time the good fundoscopic view and capture a photo is a skill, that may take longer to acquire than performing a direct fundoscopy [15].

In 2001 PanOptic (Welch Allyn Inc., Skaneateles Falls, New York, USA) introduced its new ophthalmoscope and later developed an FDA approved smartphone adapter called iExaminer[4, 15, 17]. The adapter is suitable only for iPhone 6S, which is an outdated model and unfortunately does not provide satisfactory image quality. Therefore, we designed our own adapter which is suitable for any smartphone. Thus, it does not have to be redesigned with each new generation of smartphones. As of the time of this writing, the adapter is not FDA approved and is meant to be used only in research settings. Current smartphones can take high-quality video recordings, which allows to rewind the video and take a snapshot of the best frame for storage. It also allows assessing the venous pulsation. What is more exciting, 
microscopic eye movements allow taking snapshots of two side-by-side photos for 3D reconstruction and stereoscopic image viewing, which is the next level of optic disc assessment (Figure 4 a-c).

In our study image acquisition was successful in all patients, even those who were uncooperative or had depressed level of consciousness, mainly due to utilization of video recording and ability to use less light intensity thanks to high-quality of current smartphone cameras. There was a steep learning curve for examining difficult patients, which sometimes required an assistant to keep eyelids open.

Multiple studies have shown that medical students and junior residents prefer using fundus photography over direct ophthalmoscopy. $[5,10]$ In our experience, medical students rotating with the neurology department were able to take high-quality fundus videographs after only 15-20 minutes of demonstration and practice.

Furthermore, using a smartphone-powered light source, we eliminated the necessity to charge and carry the battery, reducing the number of necessary items to just the smartphone and the headpiece of Panoptic Ophthalmoscope.

Given our initial successful experience, multiple copies of the adapter were printed and distributed to all residents in the department for further evaluation. We also expanded its use to all inpatient and outpatient clinical settings. In our experience, the main limiting factor for widespread utilization of fundus video/photography among all residents was the hesitation to adopt a new exam tool and medico-legal concerns tied to image interpretation.

\section{Conclusions}

We present our initial experience of using a custom-built universal smartphone adapter for PanOptic ophthalmoscope to obtain videographs and photographs of ocular fundus in patients presenting to the emergency department with a neurological complaint. Acquisition of high-quality photos enables a highyield diagnostic tool, allows revisiting the image in patient's chart and serves as a great teaching tool. 
Improvement of smartphone cameras opens vast horizons for stereo-fundoscopy and 3D reconstruction of retinal fundus without using sophisticated and costly equipment.

\section{Declarations}

Ethics approval and consent to participate: All methods were performed in accordance with the relevant guidelines and regulations. According to Policy Number 16 outlined in "Standard Operating Policies and Procedures" of IRB Committee of the University of California, Irvine, "Quality Improvement activities designed to continuously improve the quality or performance of a department or program or health care" do not require prior IRB approval. Since the above data was collected as part of the resident quality improvement project, IRB approval was waived.

Upon admission to the hospital all patients or their legal representatives signed general Terms and Conditions agreement, which reads: "Use of medical information and specimens: I understand that my medical information, photographs and/or video in any form may be used for other UC Irvine Health purposes, such as quality improvement, patient safety and education. I also understand that my medical information and tissue, fluids, cells and other specimens (collectively, "Specimens") that UC Irvine Health may collect during the course of my treatment and care may be used and shared with researchers." Signed terms and conditions forms for all patients are available upon reasonable request. Furthermore, fundoscopy is a part of neurological exam and does not require separate written consent.

Consent for publication. Not applicable

Availability of data and materials: The datasets used and/or analyzed during the current study are available from the corresponding author on reasonable request

Competing interests: The authors declare that they have no competing interests Funding: None 
Authors' contributions: TK designed the panoptic adapter, performed fundoscopic examinations, collected and interpreted the data and was a major contributor in writing the manuscript. LM interpreted the data and was a major contributor in writing the manuscript. TM interpreted the data and was a major contributor in writing the manuscript. All authors read and approved the final manuscript.

Acknowledgements: None

\section{References}

1. Golombievski E, Doerrler MW, Ruland SD, McCoyd MA, Biller J. Frequency of direct funduscopy upon initial encounters for patients with headaches, altered mental status, and visual changes: A pilot study. Frontiers in Neurology. 2015;6 NOV:10-2.

2. Shubhakaran K, Bidot S, Bruce BB, Newman NJ, Biousse V. Nonmydriatic retinal photography in the evaluation of acute neurologic conditions. Neurology: Clinical Practice. 2014;4:94-5.

3. Ko MW, Busis NA. Tele-Neuro-Ophthalmology: Vision for 20/20 and Beyond. Journal of neuroophthalmology : the official journal of the North American Neuro-Ophthalmology Society.

2020;40:378-84.

4. Mackay DD, Garza PS, Bruce BB, Newman NJ, Biousse V. The demise of direct ophthalmoscopy. Neurology: Clinical Practice. 2015;5:150-7.

5. Pérez MA, Bruce BB, Newman NJ, Biousse V. The use of retinal photography in nonophthalmic settings and its potential for neurology. Neurologist. 2012;18:350-5.

6. Irani NK, Bidot S, Peragallo JH, Esper GJ, Newman NJ, Biousse V. Feasibility of a Nonmydriatic Ocular Fundus Camera in an Outpatient Neurology Clinic. Neurologist. 2020;25:19-23.

7. Bruce BB, Biousse V, Newman NJ. Nonmydriatic ocular fundus photography in neurologic emergencies. JAMA Neurology. 2015;72:455-9.

8. Mackay DD, Garza PS. Ocular Fundus Photography as an Educational Tool. Seminars in Neurology. 2015;35:496-505.

9. Bruce BB, Newman NJ, Pérez MA, Biousse V. Non-mydriatic ocular fundus photography and telemedicine: Past, present, and future. Neuro-Ophthalmology. 2013;37:51-7.

10. Biousse V, Bruce BB, Newman NJ. Ophthalmoscopy in the 21st century: The 2017 H. Houston Merritt Lecture. Neurology. 2018;90:167-75.

11. Alm M, Hautala N, Bloigu R, Huhtakangas J. Comparison of optic disc evaluation methods in neurology emergency patients. Acta Neurologica Scandinavica. 2019;140:449-51.

12. Thulasi P, Fraser CL, Biousse V, Wright DW, Newman NJ, Bruce BB. Nonmydriatic ocular fundus photography among headache patients in an emergency department. Neurology. 2013;80:432-7. 13. Dunn HP, Teo KZ, Smyth JWP, Weerasinghe LS, Costello J, Pampapathi P, et al. Using non-mydriatic fundus photography to detect fundus pathology in Australian metropolitan emergency departments: A prospective prevalence and diagnostic accuracy study. EMA - Emergency Medicine Australasia. 2021;33:302-9.

14. Bruce BB, Lamirel C, Biousse V, Ward A, Heilpern KL, Newman NJ, et al. Feasibility of nonmydriatic ocular fundus photography in the emergency department: Phase i of the FOTO-ED study. Academic Emergency Medicine. 2011;18:928-33. 
15. Zafar S, Cardenas YM, Leishangthem L, Yaddanapudi S. Opinion and special articles: Amateur fundus photography with various new devices Our experience as neurology residents. Neurology. 2018;90:897-901.

16. Laurent C, Hong SC, Cheyne KR, Ogbuehi KC. The detection of spontaneous venous pulsation with smartphone video ophthalmoscopy. Clinical Ophthalmology. 2020;14:331-7.

17. Rodenbeck SJ, MacKay DD. Examining the ocular fundus in neurology. Current Opinion in Neurology. 2019;32:105-10. 


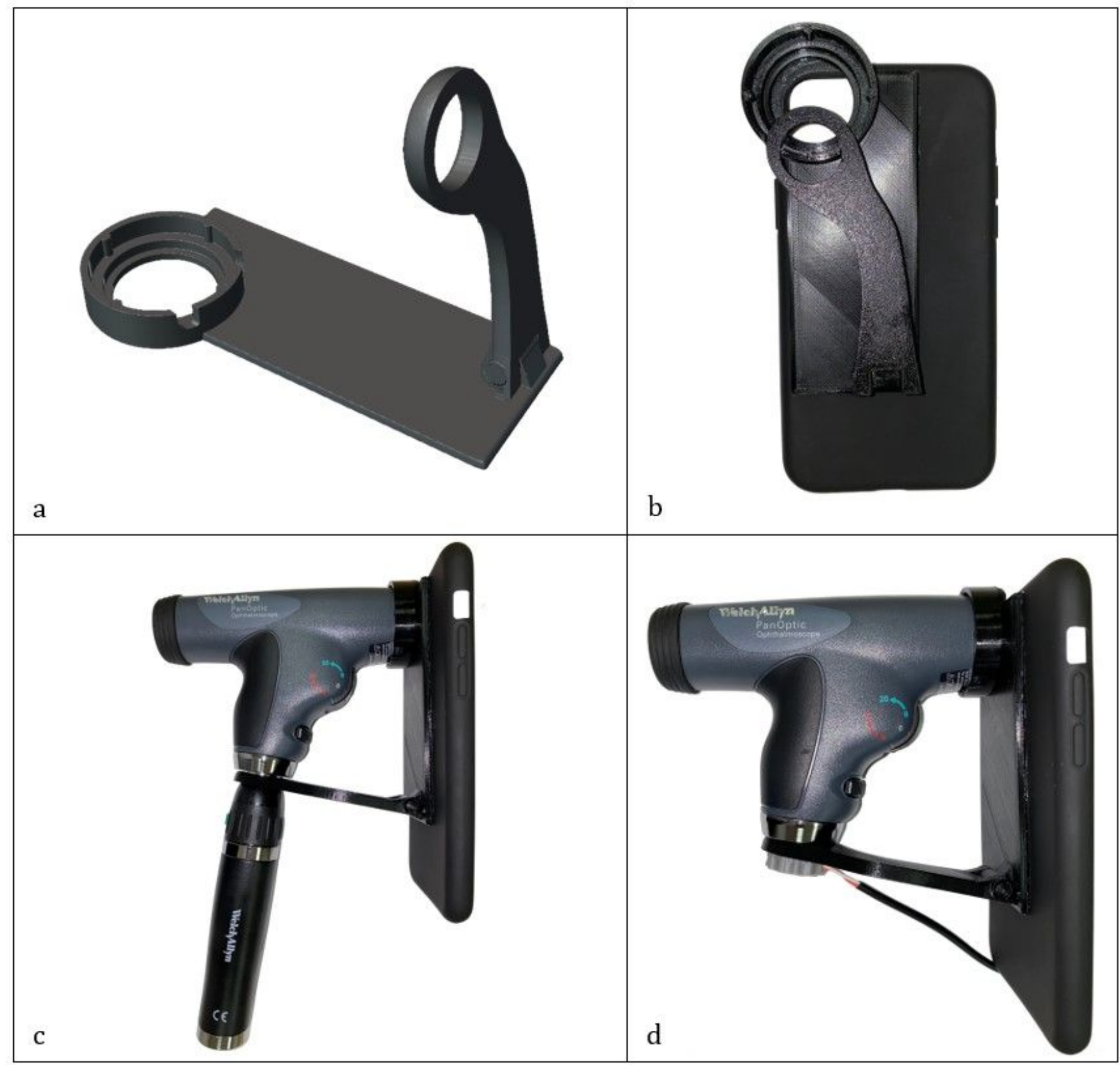

Figure 1: Universal smartphone adapter for Panoptic ophthalmoscope. a: 3D model of the adapter. b: The adapter is permanently attached to the smartphone case. c: Assembled ophthalmoscope with factory battery. $d$ : assembled ophthalmoscope with smartphone-powered light source.

\section{Figure 1}

"See image above for figure legend" 
Figure 2: Fundoscopic abnormalities and incidental findings.

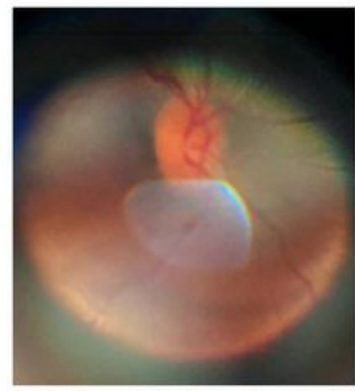

a. Incidental finding of a small optic disc in a 57-years-old woman with blurry vision and migraine attack.

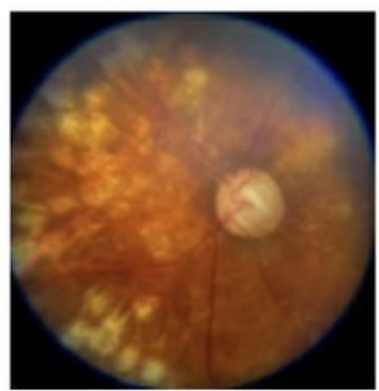

d. Multiple laser ablation scars in a 76years-old man with blurred vision, fixed and dilated pupil, who was found to have acute angle closure glaucoma.

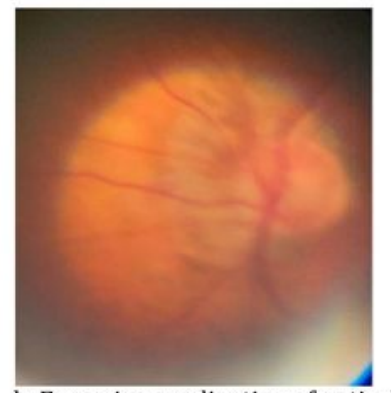

b. Excessive myelination of optic disc in an 89-years-old woman with binocular diplopia of unclear etiology

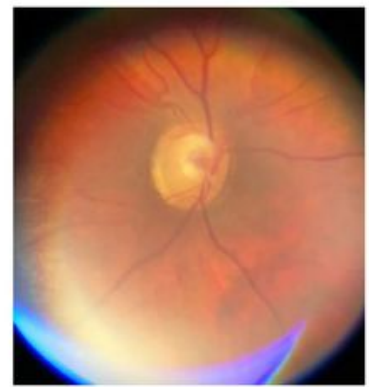

c. Optic disc pallor in a 30-years-old woman with vision, who was subsequently diagnosed with Neuromyelitis Optica

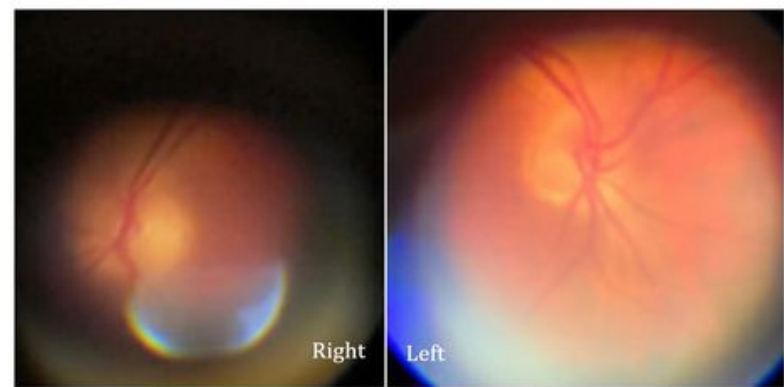

e. Bilateral grade 2 papilledema in a 55-years-old with Anaplastic ependymoma
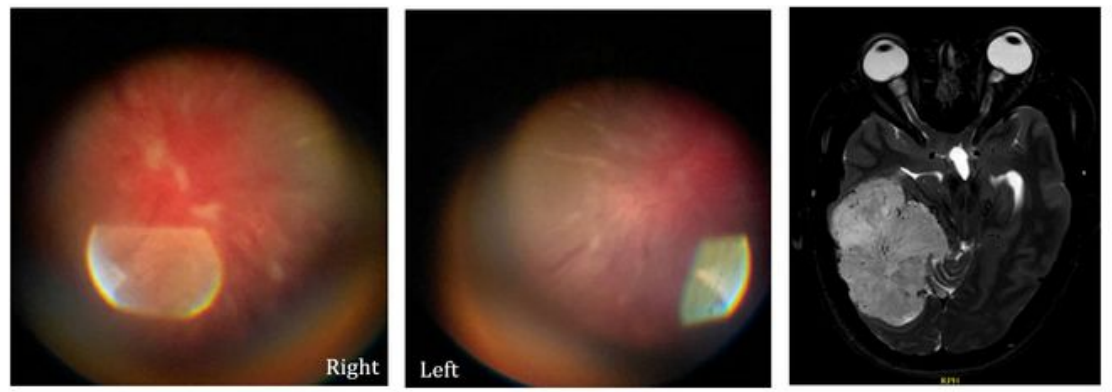

f. Bilateral grade 4 papilledema in a 40 -years-old woman with blurry vision, who was found to have a large right tentorial meningioma.
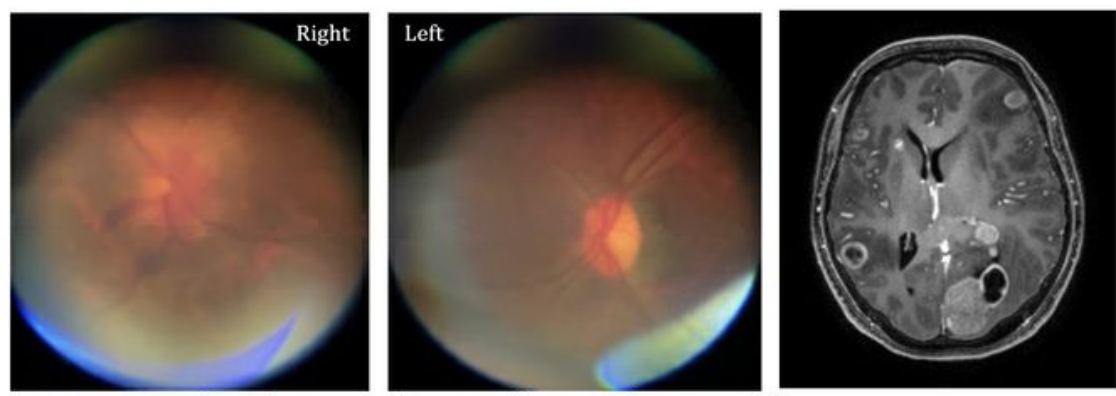

g. Foster-Kennedy syndrome in a 50-years-old man with metastatic lung cancer

\section{Figure 2}

\section{"See image above for figure legend"}




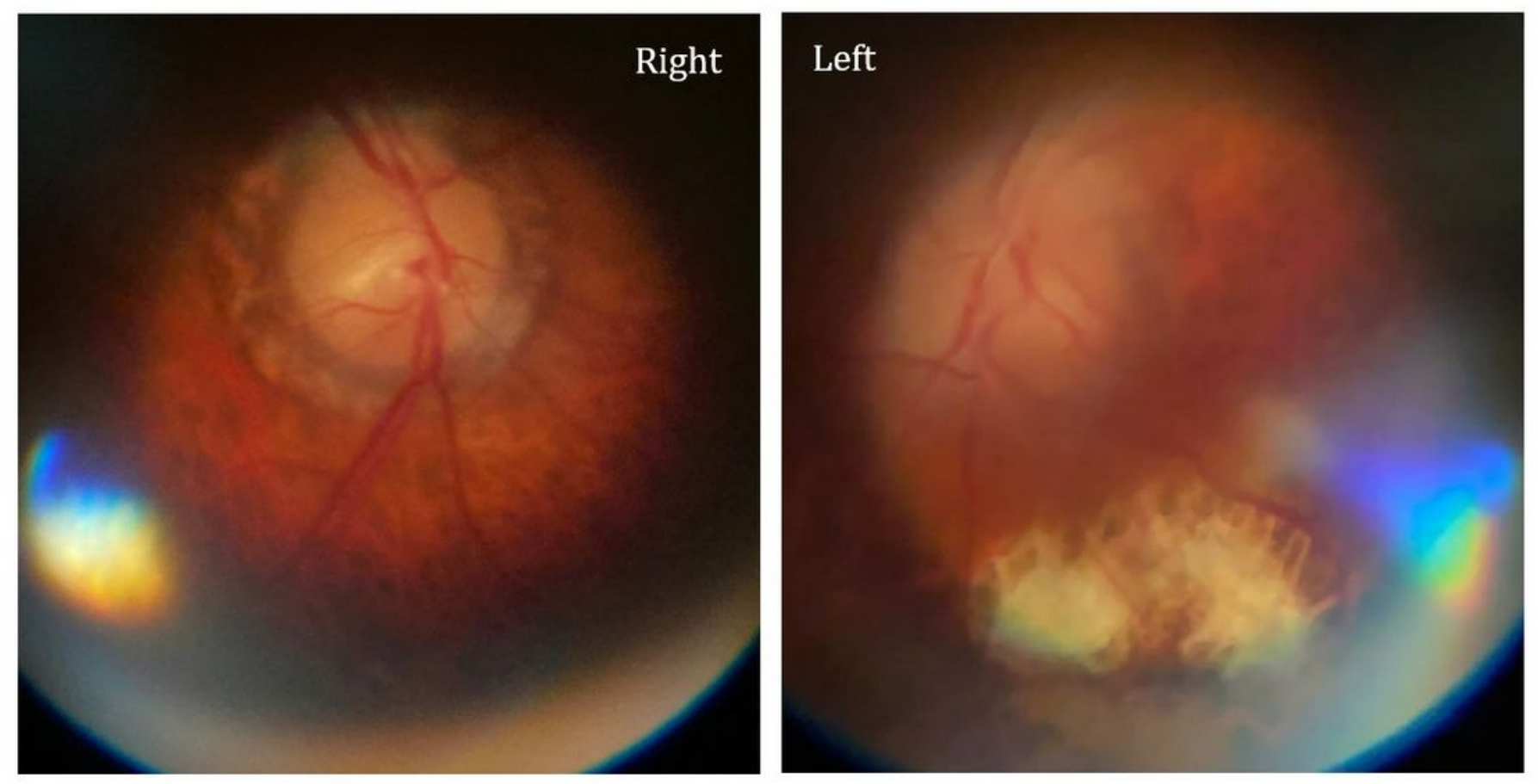

Figure 3. Bilateral grade 2 papilledema on a 78-years-old patient with leptomeningeal carcinomatosis

Figure 3

"See image above for figure legend" 

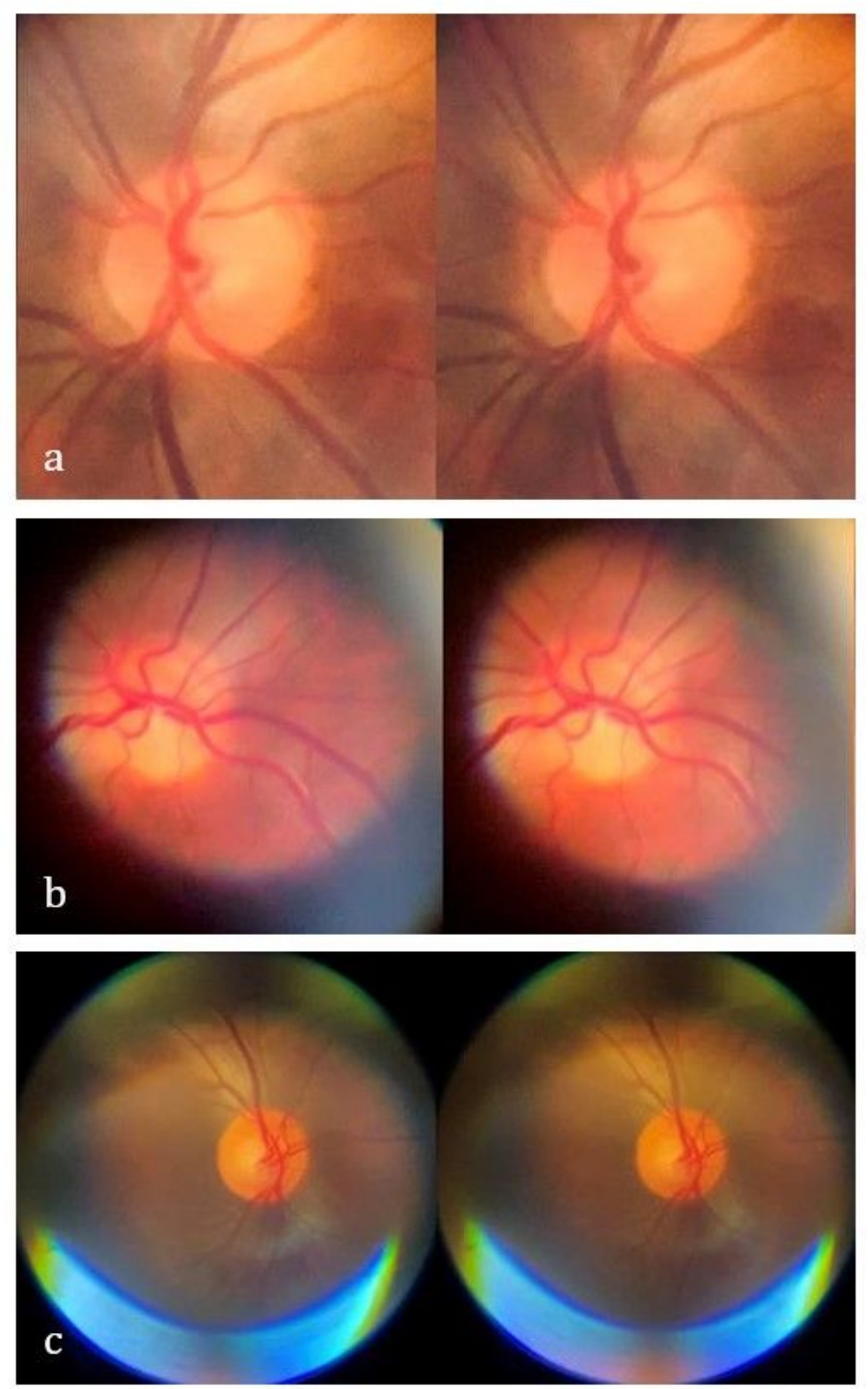

Figure 4. Stereoscopic images of the ocular fundi generated by capturing two consecutive frames from a video recording after subtle eye movement (requires google cardboard or similar for stereoscopic viewing).

\section{Figure 4}

"See image above for figure legend" 